\title{
A Stimulating Concept: Bioelectronic Medicine in Inflammatory Disease
}

\author{
Peder S Olofsson \\ Department of Biomedical Sciences, The Feinstein Institute for Medical Research, Manhasset, New York, United States of America
}

\begin{abstract}
Recent discoveries in neuroscience and immunology have revealed that stimulation of specific neural circuits can ameliorate certain experimental inflammatory diseases. Accumulating evidence on a prototypical reflex circuit that includes the vagus nerve, termed the "inflammatory reflex," has spawned clinical trials using implanted vagus nerve stimulators to treat patients with chronic inflammatory diseases. Modulation of neural reflex activity potentially represents a groundbreaking advance in treatment options for chronic inflammation, but our understanding of the involved components and signals is still incomplete. Here, the experimental basis for use of bioelectronic medicine in treatment of inflammatory disease and future challenges in this rapidly evolving field are reviewed and discussed.
\end{abstract}

Online address: www.bioelecmed.org

doi: 10.15424/bioelectronmed.2014.00007

\section{INTRODUCTION}

Inflammation is important for microbial defense and tissue healing, but it must be very carefully managed to maintain homeostasis and health. Excessive and nonresolving inflammation disrupts homeostasis and may cause inflammatory diseases. Such immune system dysregulation underlies septic shock, rheumatoid arthritis, inflammatory bowel disease, psoriasis, multiple sclerosis, atherosclerosis, certain cancers and a range of other diseases. Consequently, nonresolving inflammation contributes significantly to morbidity and mortality worldwide $(1,2)$.

Steroidal and nonsteroidal antiinflammatory drugs, small molecule compounds and specific anticytokine drugs are widely used to treat inflammatory diseases. However, this therapy may cause serious side effects and yet fail to provide satisfactory help for a signifi- cant portion of patients. Development of effective treatment to regulate specific inflammatory processes with limited side effects has proved challenging, and conceptual innovations are greatly needed $(1,2)$. Bioelectronic medicine, the interdisciplinary field that brings together molecular medicine, neuroscience and bioengineering, holds great promise for targeted and specific therapy in the near future and may have utility in treatment of inflammatory diseases, for example, by modulating signals in neural reflexes (3-5).

\section{NEURAL REFLEX CIRCUITS ARE AN INTEGRAL COMPONENT OF THE IMMUNE SYSTEM}

Neural reflex circuits are crucial regulators of organ function and homeostasis. For example, heart rate, blood pressure and body temperature are closely moni-

Address correspondence to Peder S Olofsson, The Feinstein Institute for Medical Research, 350 Community Drive, Manhasset, NY 11030. Phone: 516-562-1 127; Fax: 516-562-2356; E-mail: Peder.Olofsson@ki.se.

Submitted September 17, 2014; Accepted for publication September 17, 2014; Published Online (www.bioelecmed.org) December 2, 2014.

\section{The Feinstein Institute for Medical Research} Empowering Imagination. Pioneering Discovery.

tored and regulated by neural signals. Interestingly, a connection also between the nervous system and inflamed tissue was described when the four clinical hallmarks of inflammation redness, swelling, warmth and pain were defined by Aulus Cornelius Celsus in De Medicina. It has now become clear that body surfaces are equipped with a dense mesh of sensory nerves that possess similar molecular recognition systems for danger signals as immune cells do (6). Invading bacteria directly activate sensory nerves and elicit axonal signals (7). Endotoxin and proinflammatory cytokines also elicit sensory signals that result in a motor nerve response (8-10). In this way, neural reflexes can respond to microbial invasion and tissue injury.

Recent insights into these mechanisms have fundamentally changed our understanding of immune system homeostasis: The immune system can no longer be regarded fully autonomous, because it is regulated by homeostatic autonomic neural reflexes. These discoveries are now spawning novel and revolutionary ideas for support of tissue healing and treatment of chronic inflammatory diseases using electrical nerve stimulation to regulate inflammation (11-15). Furthermore, the insight that sensory nerve signals arise as a result of microbial inva- 
sion and immune activity suggest that it may be possible to monitor inflammation by measuring signals in the nervous system.

\section{A PROTOTYPICAL IMMUNE- REGULATORY NEURAL CIRCUIT: THE INFLAMMATORY REFLEX}

A particular immune-regulatory neural circuit, in which the vagus nerve plays a key role, is called the "inflammatory reflex" and has been the focus of extensive study over the last decade $(11,15,16)$. In this reflex circuit, vagus nerve fibers report inflammatory events in its sensory domain to the brainstem, which elicits regulating motor signals to the periphery. For example, IL-1 $\beta$ injected intraperitoneally or in the portal vein activates sensory neurons and signals in the vagus nerve that ultimately give rise to fever (17) and triggers motor signals in the splenic nerve $(8,15,18)$. Electrical stimulation of the vagus nerve (VNS) inhibits release of proinflammatory cytokines by activating $\alpha 7$ nicotinic acetylcholine receptors $(\alpha 7 \mathrm{nAChR})$ on leukocytes in spleen $(19,20)$.

Cholinergic signals in the vagus nerve travel to the celiac ganglion and are propagated to the spleen via the adrenergic splenic nerve, which terminates in close apposition of choline acetyltransferase (ChAT)-expressing lymphocytes. A specific $\mathrm{ChAT}^{+}$lymphocyte subset, $\mathrm{CD} 4^{+}$ $\mathrm{CD}_{4} 4^{\text {hi }} \mathrm{CD} 2 \mathrm{~L}^{\text {low }} \mathrm{ChAT}^{+} \mathrm{T}$ cells, responds to adrenergic signals with release of acetylcholine, the cognate ligand for the $\alpha 7 \mathrm{nAChR}$, and relays the splenic nerve signals to $\alpha 7 \mathrm{nAChR}$-expressing innate immune cells (20-27). This complex circuit of sensory and motor nerve fibers and immune cells that relay neural signals represents an autonomic reflex that senses and modulates cytokine production and inflammation $(14,16,28-30)$.

\section{BIOELECTRONIC INTERVENTION IN THE INFLAMMATORY REFLEX}

Because mechanistic details of the inflammatory reflex are known, it has been possible to investigate whether activation of circuit components, including electrical nerve stimulation, can treat inflammatory disease. Cervical VNS improved survival and reduced organ damage in animal models of severe sepsis $(23,31,32)$, ischemia-reperfusion $(33,34)$, artery occlusion shock (35), ventilator-induced lung injury (36), hemorrhagic shock (37) and burn injury (38). In a model of peritonitis, signals in the vagus nerve controlled resolution and proresolving mediators of inflammation (39). VNS also reduced disease severity in experimental models of rheumatoid arthritis (40), colitis (41,42), arthritis (43) and postoperative ileus $(44,45)$.

Based on this knowledge, implanted vagus nerve stimulators are now used in exploratory clinical trials to treat rheumatoid arthritis, colitis and postoperative ileus. Preliminary observations reported from the rheumatoid arthritis clinical trial by Frieda Koopman et al. indicate reduced disease scores and reduced levels of C-reactive protein in blood after a brief daily activation of an implanted vagus nerve stimulator. Reported side effects were limited and benign (46). Although definitive data is pending, these first observations of the effect of implanted vagus nerve stimulators are quite encouraging.

\section{A MULTITUDE OF NEURAL CIRCUITS REGULATE INFLAMMATION}

In addition to the inflammatory reflex, a number of other neural pathways regulate inflammation and immunity. Activation of muscarinic signaling in the central nervous system mimics the effect of direct vagus nerve stimulation on cytokine release in endotoxemia and requires an intact vagus nerve (47-49). Signals in the vagus nerve can also inhibit intestinal cytokine release independently of the spleen (44), bypassing the prototypical signaling pathway in the inflammatory reflex. Furthermore, electrical stimulation applied to the sciatic nerve regulates innate immune activation by controlling vagus nervedependent dopamine release from the adrenal gland (50,51). Another specific neural immune reflex acts as a gateway for entry of autoreactive T cells to the CNS. In this circuit, sensory signals that arise in the hind limb reach the spinal cord and brain stem to activate adrenergic neurons that regulate T cell extravasation by modulating the expression of chemokine receptors on vascular endothelial cells (52). Yet another neural pathway regulates hepatic invariant NKT cells (iNKT) cell activity through an adrenergic signal, and blockade of this neural conduit prevents stroke-associated pneumonia in mice (53). Endotoxin and IL-1 $\beta$ trigger activity in the hypothalamus-pituitary-adrenal axis with a resulting increase in blood levels of glucocorticoids (54), which have antiinflammatory properties. This list of immune-regulatory neural circuitry is not exhaustive, and it is very likely that many similar and related autonomic reflex circuits remain to be discovered. Presently, there is sufficient data to conclude that neural signals exert significant control over the immune response to trauma and microbial invasion. Modulation of nervous signals can alter the course of inflammation, but much remains to be discovered on the details of the intricate signals involved and how to modulate the neural circuitry to achieve optimal control.

\section{BIOELECTRONIC MEDICINE BEYOND INFLAMMATION}

The scope of bioelectronic medicine is not limited to treatment of chronic inflammation. For example, electrical VNS reduces bleeding time and blood loss in experimental traumatic injury (55). Burn injury victims often suffer from fluid leakage from blood vessels and complications from severe tissue edema. Electrical VNS reduced vascular permeability in the skin and lung in an animal model of burn injury, significantly reducing tissue edema in treated animals (56). VNS had beneficial effects in experimental heart failure and is currently under investigation for treatment of heart failure in humans $(57,58)$. Stimulation of peripheral nerves, including VNS, is also used in treatment of intractable epilepsy and certain forms of headaches and migraine (59). Application of a local DC current may be helpful in rehabilitation after 
stroke and in other diseases with damage to the central nervous system (60). Moreover, bioelectronic medicine is used experimentally to restore damaged vision (61). In light of this, a number of diseases considered to primarily be of noninflammatory origin may significantly benefit from targeted nerve stimulation using bioelectronic devices. Furthermore, as discussed elsewhere in this issue of Bioelectronic Medicine, implantable sensors for a range of physiological variables are being developed (62). Coupled with active bioelectronic devices, these technologies have the potential to revolutionize diagnosis and treatment by recording and modulating signals in real time. Currently available devices have, however, not yet been deemed sufficiently reliable for patient use without human supervision (63).

\section{CURRENT CHALLENGES IN BIOELECTRONIC MEDICINE}

The optimal signaling in the inflammatory reflex (and other reflex circuits) for maximum effect on the immune response is not known. The precise identity of the involved neurons remains unclear and the effects on inflammation and immune cell activity of specific stimulation modalities have not been determined. To study this in detail, new stimulation techniques with better signal control, ideally connecting to individual nerve fibers, would be of great utility.

The role of the sensory portion of the inflammatory reflex for immune system regulation is not well understood. It is possible that select afferent signals in the vagus nerve activate motor centers in the brain that in turn regulate immune cell behavior through signals in the efferent vagus nerve, and through other efferent neural and hormonal mechanisms. The recent discovery that sensory nerves can directly detect presence of pathogens and regulate local immune responses $(6,7,10)$ inspires a range of new opportunities. Decoding the afferent signals elicited by tissue injury, pathogen invasion, cytokines and inflammation will be an important step in understanding the reflex control of immune homeostasis. This may be significant, because it is conceivable that sensory signals can be interpreted and used to guide real-time diagnosis and bioelectronic modulation of ongoing inflammation.

Input to bioelectronic devices need not be limited to neural signals. As a multitude of measurements become accessible in real time, it will be a challenge to integrate the complex sets of information in a meaningful way to make it useful for improving therapy.

High biocompatibililty is naturally vital for maintaining the integrity of the tissue-machine interface (63-66) and necessary to reduce the risk for undesirable side effects from implanted devices. An alternative is development of external devices that use other methods to activate neural circuits, for example magnetic fields. If the future clinical trials show efficacy of nerve stimulation in treatment of inflammatory diseases, such noninvasive approaches may be well worth intense pursuit.

\section{CONCLUSION}

We are just beginning to understand some of the molecular mechanisms that govern the multidirectional communication between damaged tissue, microbes, immune cells and the nervous system. Sensory nerves detect microbial invasion and report on immune system activity. Motor nerves regulate cytokine release and inflammation. The discovery of the inflammatory reflex in the control of immune homeostasis and the promising, yet preliminary, results from studies of implanted devices for treatment of inflammatory disease are truly of breakthrough character. Although bioelectronic medicine is in its infancy, it has the potential to revolutionize diagnosis and treatment of a wide range of inflammatory diseases.

\section{ACKNOWLEDGMENTS}

This work was supported by funding from Svenska Läkaresällskapet. I thank Valentin A Pavlov, Kevin J Tracey and LaQueta K Hudson for helpful comments.

\section{DISCLOSURE}

The author declares that he has no competing interests as defined by Bioelectronic Medicine, or other interests that might be perceived to influence the results and discussion reported in this paper.

\section{REFERENCES}

1. Cotran R, Kumar V, Collins T (eds.). (1999) Robbins Pathologic Basis of Disease. 6th edition. Philadelphia: W.B. Saunders. 1424 pp.

2. Nathan C, Ding A. (2010) Nonresolving inflammation. Cell. 140:871-82.

3. Birmingham K, et al. (2014) Bioelectronic medicines: a research roadmap. Nat. Rev. Drug Discov. 13:399-400.

4. Famm K, Litt B, Tracey KJ, Boyden ES, Slaoui M. (2013) Drug discovery: a jump-start for electroceuticals. Nature. 496:159-61.

5. Reardon S. (2014) Electroceuticals spark interest. Nature. 511:18.

6. Chiu IM, von Hehn CA, Woolf CJ. (2012) Neurogenic inflammation and the peripheral nervous system in host defense and immunopathology. Nat. Neurosci. 15:1063-7.

7. Chiu IM, et al. (2013) Bacteria activate sensory neurons that modulate pain and inflammation. Nature. 501:52-7.

8. Niijima A. (1996) The afferent discharges from sensors for interleukin 1 beta in the hepatoportal system in the anesthetized rat. J. Auton. Nerv. Syst. 61:287-91.

9. Andersson U, Tracey KJ. (2012) Neural reflexes in inflammation and immunity. J. Exp. Med. 209:1057-68

10. Riol-Blanco L, et al. (2014) Nociceptive sensory neurons drive interleukin-23-mediated psoriasiform skin inflammation. Nature. 510:157-61.

11. Andersson U, Tracey KJ. (2012) Reflex principles of immunological homeostasis. Annu. Rev. Immunol. 30:313-35.

12. Zitnik RJ. (2011) Treatment of chronic inflammatory diseases with implantable medical devices. Cleve. Clin. J. Med. 78 Suppl 1:S30-4.

13. Koopman FA, et al. (2011) Restoring the balance of the autonomic nervous system as an innovative approach to the treatment of rheumatoid arthritis. Mol. Med. 17:937-48.

14. Boeckxstaens G. (2013) The clinical importance of the anti-inflammatory vagovagal reflex. Handb. Clin. Neurol. 117:119-34.

15. Olofsson PS, Rosas-Ballina M, Levine YA, Tracey KJ. (2012) Rethinking inflammation: Neural circuits in the regulation of immunity. Immunol. Rev. 248:188-204.

16. Tracey KJ. (2002) The inflammatory reflex. $N a$ ture. 420:853-9.

17. Watkins LR, et al. (1995) Blockade of interleukin-1 induced hyperthermia by subdiaphragmatic vagotomy: evidence for vagal mediation of immunebrain communication. Neurosci. Lett. 183:27-31.

18. Pavlov VA, Tracey KJ. (2012) The vagus nerve 
and the inflammatory reflex-linking immunity and metabolism. Nat. Rev. Endocrinol. 8:743-54.

19. Wang $H$, et al. (2003) Nicotinic acetylcholine receptor alpha7 subunit is an essential regulator of inflammation. Nature. 421:384-8.

20. Olofsson PS, et al. (2012) alpha7 nicotinic acetylcholine receptor (alpha7nAChR) expression in bone marrow-derived non-T cells is required for the inflammatory reflex. Mol. Med. 18:539-43.

21. Rosas-Ballina M, et al. (2008) Splenic nerve is required for cholinergic antiinflammatory pathway control of TNF in endotoxemia. Proc. Nat. Acad. Sci. U. S. A. 105:11008-13.

22. Rosas-Ballina M, et al. (2011) Acetylcholine-synthesizing $\mathrm{T}$ cells relay neural signals in a vagus nerve circuit. Science. 334:98-101.

23. Pavlov VA, et al. (2007) Selective alpha7-nicotinic acetylcholine receptor agonist GTS-21 improves survival in murine endotoxemia and severe sepsis. Crit. Care Med. 35:1139-44.

24. Rosas-Ballina M, et al. (2009) The selective alpha7 agonist GTS-21 attenuates cytokine production in human whole blood and human monocytes activated by ligands for TLR2, TLR3, TLR4, TLR9, and RAGE. Mol. Med. 15:195-202.

25. Bellinger DL, Lorton D, Hamill RW, Felten SY, Felten DL. (1993) Acetylcholinesterase staining and choline acetyltransferase activity in the young adult rat spleen: lack of evidence for cholinergic innervation. Brain. Behav. Immun. 7:191-204.

26. Lu B, et al. (2014) Alpha 7 nicotinic acetylcholine receptor signaling inhibits inflammasome activation by preventing mitochondrial DNA release. Mol. Med. 2014 May 13. [Epub ahead of print].

27. Huston JM, et al. (2006) Splenectomy inactivates the cholinergic antiinflammatory pathway during lethal endotoxemia and polymicrobial sepsis. J. Exp. Med. 203:1623-8.

28. Borovikova LV, et al. (2000) Vagus nerve stimulation attenuates the systemic inflammatory response to endotoxin. Nature. 405:458-62.

29. Sternberg EM. (2006) Neural regulation of innate immunity: a coordinated nonspecific host response to pathogens. Nat. Rev. Immunol. 6:318-28.

30. Sundman E, Olofsson PS. (2014) Neural control of the immune system. Adv. Physiol. Educ. 38:135-9.

31. Huston JM, et al. (2007) Transcutaneous vagus nerve stimulation reduces serum high mobility group box 1 levels and improves survival in murine sepsis. Crit. Care Med. 35:2762-8.

32. Boland C, et al. (2011) Electrical vagus nerve stimulation and nicotine effects in peritonitisinduced acute lung injury in rats. Inflammation. 34:29-35.

33. Bernik TR, et al. (2002) Cholinergic antiinflammatory pathway inhibition of tumor necrosis factor during ischemia reperfusion. J. Vasc. Surg. 36:1231-6.

34. Mioni C, et al. (2005) Activation of an efferent cholinergic pathway produces strong protection against myocardial ischemia/reperfusion injury in rats. Crit. Care Med. 33:2621-8.

35. Altavilla D, et al. (2006) Activation of the cholin- ergic anti-inflammatory pathway reduces NFkappaB activation, blunts TNF-alpha production, and protects against splanchnic artery occlusion shock. Shock. 25:500-6.

36. Bregeon F, et al. (2011) Activation of nicotinic cholinergic receptors prevents ventilator-induced lung injury in rats. PLOS ONE. 6:e22386.

37. Guarini S, et al. (2003) Efferent vagal fibre stimulation blunts nuclear factor-kappaB activation and protects against hypovolemic hemorrhagic shock. Circulation. 107:1189-94.

38. Song XM, et al. (2010) Effect of vagus nerve stimulation on thermal injury in rats. Burns. 36:75-81.

39. Mirakaj V, Dalli J, Granja T, Rosenberger P, Serhan CN. (2014) Vagus nerve controls resolution and pro-resolving mediators of inflammation. J. Exp. Med. 211:1037-48.

40. Levine YA, et al. (2014) Neurostimulation of the cholinergic anti-inflammatory pathway ameliorates disease in rat collagen-induced arthritis. PLoS One. 9:e104530.

41. Meregnani J, et al. (2011) Anti-inflammatory effect of vagus nerve stimulation in a rat model of inflammatory bowel disease. Auton. Neurosci. 160:82-9.

42. Ji H, et al. (2014) Central cholinergic activation of a vagus nerve-to-spleen circuit alleviates experimental colitis. Mucosal Immunol. 7:335-47.

43. Zhang P, Han D, Tang T, Zhang X, Dai K. (2008) Inhibition of the development of collagen-induced arthritis in Wistar rats through vagus nerve suspension: a 3-month observation. Inflamm. Res. 57:322-8.

44. Matteoli $G$, et al. (2013) A distinct vagal antiinflammatory pathway modulates intestinal muscularis resident macrophages independent of the spleen. Gut. 63:938-48.

45. de Jonge WJ, et al. (2005) Stimulation of the vagus nerve attenuates macrophage activation by activating the Jak2-STAT3 signaling pathway. Nat. Immunol. 6:844-51.

46. Koopman FA, et al. (2012) Pilot study of stimulation of the cholinergic anti-inflammatory pathway with an implantable vagus nerve stimulation device in patients with rheumatoid arthritis (Abstract). Arthritis Rheum. 64:S195.

47. Bernik TR, et al. (2002) Pharmacological stimulation of the cholinergic antiinflammatory pathway. J. Exp. Med. 195:781-8.

48. Pavlov VA, et al. (2006) Central muscarinic cholinergic regulation of the systemic inflammatory response during endotoxemia. Proc. Natl. Acad. Sci. U. S. A. 103:5219-23.

49. Pavlov VA, et al. (2009) Brain acetylcholinesterase activity controls systemic cytokine levels through the cholinergic anti-inflammatory pathway. Brain Behav. Immun. 23:41-5.

50. Torres-Rosas R, et al. (2014) Dopamine mediates vagal modulation of the immune system by electroacupuncture. Nat. Med. 20:291-5.

51. Chavan SS, Tracey KJ. (2014) Regulating innate immunity with dopamine and electroacupuncture. Nat. Med. 20:239-41.

52. Arima Y, et al. (2012) Regional neural activation defines a gateway for autoreactive $\mathrm{T}$ cells to cross the blood-brain barrier. Cell. 148:447-57.

53. Wong CH, Jenne CN, Lee WY, Leger C, Kubes P. (2011) Functional innervation of hepatic iNKT cells is immunosuppressive following stroke. Science. 334:101-5.

54. Besedovsky H, del Rey A, Sorkin E, Dinarello CA. (1986) Immunoregulatory feedback between interleukin-1 and glucocorticoid hormones. Science. 233:652-4.

55. Czura CJ, et al. (2010) Vagus nerve stimulation regulates hemostasis in swine. Shock. 33:608-13.

56. Ortiz-Pomales YT, Krzyzaniak M, Coimbra R, Baird A, Eliceiri BP. (2013) Vagus nerve stimulation blocks vascular permeability following burn in both local and distal sites. Burns. 39:68-75.

57. Klein HU, Ferrari GM. (2010) Vagus nerve stimulation: A new approach to reduce heart failure. Cardiol. J. 17:638-44.

58. Li M, et al. (2004) Vagal nerve stimulation markedly improves long-term survival after chronic heart failure in rats. Circulation. 109:120-4.

59. Jenkins B, Tepper SJ. (2011) Neurostimulation for primary headache disorders, part 1: pathophysiology and anatomy, history of neuromodulation in headache treatment, and review of peripheral neuromodulation in primary headaches. Headache. 51:1254-66.

60. Schlaug G, Renga V, Nair D. (2008) Transcranial direct current stimulation in stroke recovery. Arch. Neurol. 65:1571-6.

61. Nirenberg S, Pandarinath C. (2012) Retinal prosthetic strategy with the capacity to restore normal vision. Proc. Natl. Acad. Sci. U. S. A. 109:15012-7.

62. Li C, Narayan RK. (2014) Monitoring the injured brain. Bioelectron. Med. 1:4-8

63. Turner AP. (2013) Biosensors: sense and sensibility. Chem. Soc. Rev. 42:3184-96.

64. Wallace GG, Moulton SE, Clark GM. (2009) Applied physics. Electrode-cellular interface. Science. 324:185-6.

65. Malliaras GG. (2013) Organic bioelectronics: a new era for organic electronics. Biochim. Biophys. Acta. 1830:4286-7.

66. Alibart F, et al. (2010) An organic nanoparticle transistor behaving as a biological spiking synapse. Adv. Funct. Mater. 20:330-7.

Cite this article as: Olofsson P. (2014) A stimulating concept: bioelectronic medicine in inflammatory disease. Bioelectron. Med. 1:30-3. 